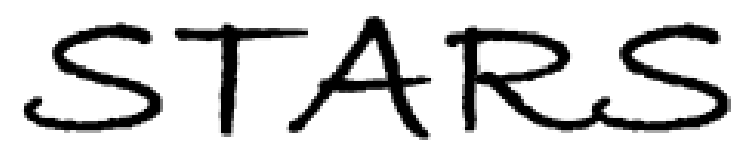

University of Central Florida

STARS

$1-1-2004$

\title{
Kapitza conductance and phonon scattering at grain boundaries by simulation
}

\author{
P. K. Schelling \\ University of Central Florida \\ S. R. Phillpot \\ P. Keblinski
}

Find similar works at: https://stars.library.ucf.edu/facultybib2000

University of Central Florida Libraries http://library.ucf.edu

This Article is brought to you for free and open access by the Faculty Bibliography at STARS. It has been accepted for inclusion in Faculty Bibliography 2000s by an authorized administrator of STARS. For more information, please contactSTARS@ucf.edu.

\section{Recommended Citation}

Schelling, P. K.; Phillpot, S. R.; and Keblinski, P., "Kapitza conductance and phonon scattering at grain boundaries by simulation" (2004). Faculty Bibliography 2000 s. 4777.

https://stars.library.ucf.edu/facultybib2000/4777

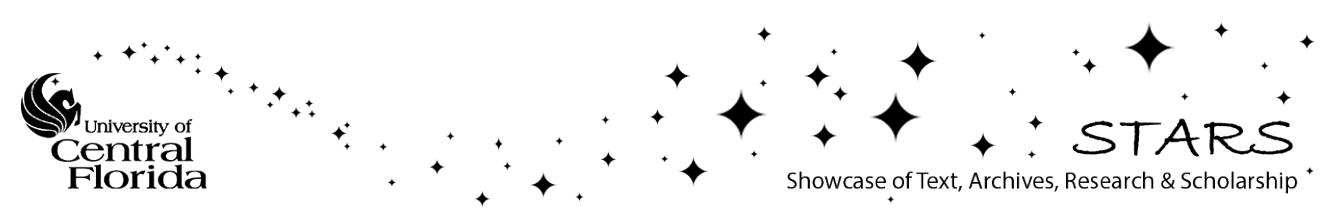




\section{Kapitza conductance and phonon scattering at grain boundaries by simulation}

Cite as: Journal of Applied Physics 95, 6082 (2004); https://doi.org/10.1063/1.1702100

Submitted: 02 February 2004 . Accepted: 18 February 2004 . Published Online: 25 May 2004

P. K. Schelling, S. R. Phillpot, and P. Keblinski

\section{ARTICLES YOU MAY BE INTERESTED IN}

Phonon wave-packet dynamics at semiconductor interfaces by molecular-dynamics simulation

Applied Physics Letters 80, 2484 (2002); https://doi.org/10.1063/1.1465106

Nanoscale thermal transport. II. 2003-2012

Applied Physics Reviews 1, 011305 (2014); https://doi.org/10.1063/1.4832615

Nanoscale thermal transport

Journal of Applied Physics 93, 793 (2003); https://doi.org/10.1063/1.1524305

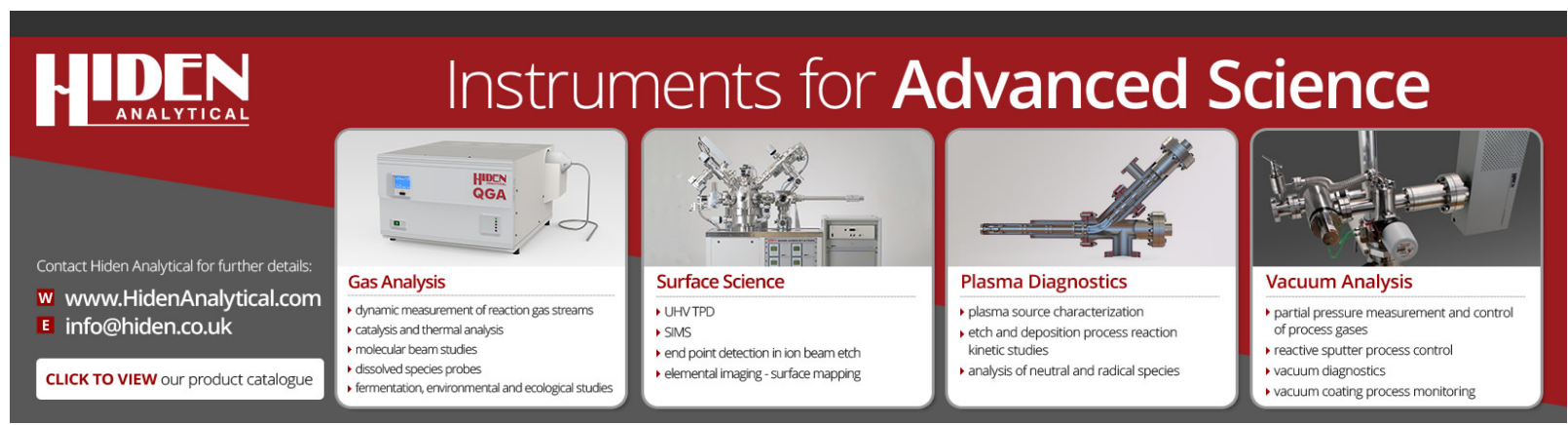




\title{
Kapitza conductance and phonon scattering at grain boundaries by simulation
}

\author{
P. K. Schellinga) \\ Materials Science Division, Argonne National Laboratory, Argonne, Illinois 60439, \\ Institute for Nanotechnology, Forschungszentrum Karlsruhe, 76021 Karlsruhe, Germany, \\ and Advanced Materials Processing and Analysis Center and Department of Physics, \\ University of Central Florida, Orlando, Florida 32816 \\ S. R. Phillpot \\ Materials Science Division, Argonne National Laboratory, Argonne, Illinois 60439 \\ and Department of Materials Science and Engineering, University of Florida, Gainesville, Florida 32611- \\ 6400 \\ P. Keblinski \\ Materials Science and Engineering Department, Rensselaer Polytechnic Institute, \\ Troy, New York 12180-3590
}

(Received 2 February 2004; accepted 18 February 2004)

\begin{abstract}
We use a nonequilibrium molecular-dynamics method to compute the Kapitza resistance of three twist grain boundaries in silicon, which we find to increase significantly with increasing grain boundary energy, i.e., with increasing structural disorder at the grain boundary. The origin of this Kapitza resistance is analyzed directly by studying the scattering of packets of lattice vibrations of well-defined polarization and frequency from the grain boundaries. We find that scattering depends strongly on the wavelength of the incident wave packet. In the case of a high-energy grain boundary, the scattering approaches the prediction of the diffuse mismatch theory at high frequencies, i.e., as the wavelength becomes comparable to the lattice parameter of the bulk crystal. We discuss the implications of our results in terms of developing a general model of scattering probabilities that can be applied to mesoscale models of heat transport in polycrystalline systems. (C) 2004 American Institute of Physics. [DOI: 10.1063/1.1702100]
\end{abstract}

\section{INTRODUCTION}

It has long been recognized that in the presence of a heat current there is a discontinuity in temperature at an interface between two different materials. ${ }^{1,2}$ As a result, interfaces possess a finite thermal conductance, $\sigma_{K}$, known as the Kapitza conductance. The relation between the thermal current $J$ and the temperature discontinuity at the interface $\Delta T$ is given as

$$
J=\sigma_{K} \Delta T .
$$

As a result of the temperature discontinuity at interfaces, a material will exhibit an effective thermal conductivity that decreases as the number of interfaces is increased. The Kapitza resistance $R_{K}=1 / \sigma_{K}$ is a measure of the resistance of an interface to the transport of heat through it.

For phonon-mediated thermal transport, the Kapitza resistance arises from the scattering of phonons at the interface. There are currently two general theoretical frameworks for understanding the origin of this interfacial resistance. The first is the acoustic mismatch model (AMM), in which the scattering of phonons at the interface arises from the difference in the acoustic impedances of the materials on the two sides. The acoustic impedance, $Z$, is the acoustic analog of

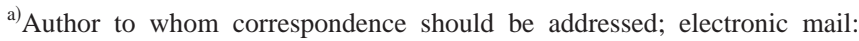
pschell@mail.ucf.edu
}

the refractive index and is simply given in terms of the speed of sound, $c$, and the density, $\rho$, as $Z=\rho c$. $^{2,3}$ The AMM is derived within continuum acoustics and thus its use becomes unjustified as the phonon wavelength approaches the lattice parameter. The key conceptual weakness of the AMM is that it does not take into account the structure of the interfaces from which the phonon scattering actually takes place. Another approach, similar in spirit to the AMM, is lattice dynamics which, since it atomistic in nature, is more appropriate for describing the scattering of phonons with wavelengths comparable to the lattice parameter. ${ }^{4,5}$ For lattice dynamics, while the structure of the boundary is considered, it is only possible to treat interfaces with a rather simple structure.

The complementary viewpoint is provided by the diffuse mismatch model (DMM). In the DMM it is assumed that all incident phonons are randomly scattered by the interface. After the scattering event, the scattered waves are emitted with an energy content proportional to the available density of states of the material on each side of the interface. ${ }^{2}$ Remarkably, for most dissimilar solid/solid interfaces the AMM and DMM result in rather similar estimates of the Kapitza conductance. Unfortunately, however, both are usually in poor agreement with the relatively few available experimental results, especially at high temperatures. ${ }^{6}$ A counterexample, however, is provided by recent data on epitaxial interfaces between TiN and single-crystal oxides $(\mathrm{MgO}$ and 
$\mathrm{Al}_{2} \mathrm{O}_{3}$ ), which seems to be consistent with the predictions of the DMM. ${ }^{6}$ Interestingly, there is essentially no difference in the thermal conductance of $\mathrm{TiN} / \mathrm{MgO}(001)$, TiN/ $\mathrm{Al}_{2} \mathrm{O}_{3}(0001)$ and TiN/MgO(111). ${ }^{6}$ Costescu, Wall, and Cahill offer two possible explanations: (i) interface scattering is so strong at all of these interfaces so that the assumptions of the DMM are satisfied, and (ii) there is so little interface disorder that phonons are hardly scattered from the interfaces at all. ${ }^{6}$

By contrast with the AMM and DMM, lattice dynamical theory does lead to different behavior at high temperatures because the phonon dispersion is naturally incorporated within this approach. Nevertheless, lattice-dynamical theory also often results in significant discrepancies with experimental results for the Kapitza conductance at interfaces between metals and dielectrics. ${ }^{7}$

In the case of grain boundaries (GBs), the situation is very different. Experimental work has so far been limited to determining the average Kapitza conductance of the GBs in a polycrystalline or nanocrystalline system. ${ }^{8,9}$ The values of the interfacial conductance determined in these studies differ very significantly: while the values determined by Yang et $a .^{9}{ }^{9}$ for yttria-stabilized zirconia are similar to the experimental values for dissimilar interfaces, the values determined by Nan and Birringer ${ }^{8}$ for a few different thermoelectric alloys are as much as four orders of magnitude smaller. There is a significant need for systematic experimental data on the thermal conductivity of both GBs and heterophase interfaces.

The theoretical situation is equally unsatisfactory. For the AMM, this is exemplified by considering the interfacial resistance of a symmetric tilt GB. A symmetric tilt GB can be thought of as being produced simply by the inversion of the stacking sequence of planes on one side of the interface with respect to the other. Thus, all of the principle crystallographic directions are aligned on the two sides. As a result, the AMM, which is based on the orientation of the elasticconstant tensors on the two sides, predicts that such an interface should have no interfacial resistance at all. This result does not seem reasonable. Indeed, molecular-dynamics (MD) simulations of thermal transport across symmetric tilt grain boundaries in $\mathrm{Si}$ have shown a significant interfacial resistance. ${ }^{10}$ The DMM seems equally unsatisfactory for such GBs since they are highly ordered and are unlikely to result in diffuse scattering. Another important theoretical approach applied to grain-boundary interfaces is to analytically compute the scattering cross section for phonons. However, these analytical calculations only account for the strain fields of the grain boundary and neglect the atomic-scale structure of the boundary. ${ }^{11}$ As a result, while the analytical results may be appropriate for long-wavelength phonons scattering from low-energy and low-angle grain boundaries, they are unlikely to apply to more general cases.

From the above discussion, it is apparent that there is no general understanding of the Kapitza conductance and of grain-boundary phonon scattering. In this article, we take the first steps towards developing a microscopic understanding of phonon scattering at interfaces and its relationship to thermal transport. We use MD simulation to directly determine the Kapitza conductance of three different twist grain bound- aries in silicon. We find that the structure and energetics of the boundary have a significant effect on the Kapitza conductance. We further analyze two of these boundaries by performing phonon wave-packet scattering simulations. This results in a more complete understanding of the dependence of phonon scattering on the phonon frequency and the grainboundary structure. Furthermore, this direct approach to the study of phonon scattering can in principle lead to models that can be used either in analytical calculations or in mesocale modeling of thermal transport.

The rest of the article is organized as follows. In Sec. II, we describe the geometry of the simulation cells used and the methodology used to determine the Kapitza conductance. We then present results for the computed Kapitza conductance for three different grain boundaries. In Sec. III we present results of wave-packet scattering from two of these boundaries. The results are compared to the predictions of the AMM and DMM. Finally, in Sec. IV we discuss our results, with a comparison to previous theoretical and experimental results. We also describe in Sec. IV how our results may be used to provide general models of interfacial scattering to be used in mesoscale models.

\section{MOLECULAR-DYNAMICS SIMULATION OF THE KAPITZA CONDUCTANCE OF SILICON GRAIN BOUNDARIES}

The thermal conductivity of a homogeneous system can be computed using an equilibrium MD simulation and the Green-Kubo formalism. ${ }^{12-15}$ However, for an inhomogeneous system, such as the GBs studied here, this approach is not appropriate, because the Kapitza conductance is a local property, which cannot be correctly treated under the assumption of the system being homogeneous. Another approach, more suitable for the study of interfacial properties, is to use a nonequilibrium MD simulation method. ${ }^{10,13,16}$ The basic idea is to create a thermal current in the MD simulation cell, and then use the resulting temperature profile to compute the thermal conductivity. For a bulk perfect crystal, the temperature gradient in the presence of a thermal current allows us to calculate the perfect-crystal thermal conductivity. ${ }^{13}$ For an interface, the temperature discontinuity $\Delta T$ at the interface can be used to compute the Kapitza conductance from Eq. (1). Thus, a nonequilibrium simulation method can be used to separately compute bulk and interfacial properties.

For this work, we use the nonequilibrium simulation method of Jund and Jullien. ${ }^{16}$ We have previously used this method to compute the thermal conductivity of perfectcrystal $\mathrm{Si}$, with a direct comparison to the results of the Green-Kubo approach. ${ }^{13}$ This approach is also very similar to that used by Maiti, Mahan, and Pantelides to compute $\sigma_{K}$ for tilt GBs in silicon. ${ }^{10}$ In Fig. 1 we show a cross section of the simulation cell. To create a thermal current along the length of the simulation, we rescale the particle velocities at each MD time step in two thin slabs each of thickness $\delta$, with the value of $\delta$ chosen to be $2 a$, where $a$ is the lattice parameter for silicon of $0.543 \mathrm{~nm}$. From one slab centered at $z=$ $-L_{z} / 4$ we add energy $\Delta \varepsilon$ at each MD time step; we remove energy $\Delta \varepsilon$ at each MD time step from the other slab centered 


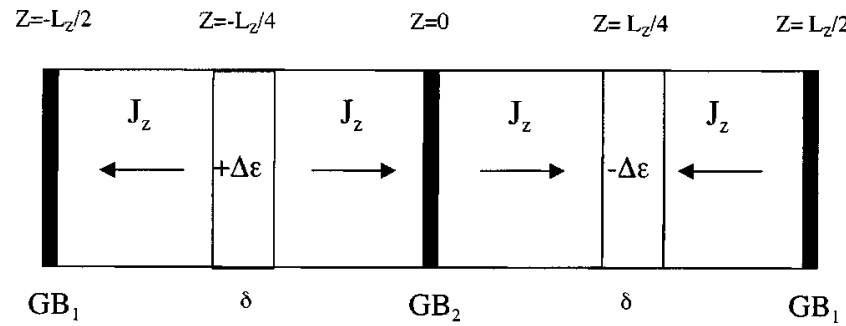

FIG. 1. Schematic representation of the three-dimensional periodic simulation cell for direct computation of the Kapitza conductance. At each MD time step, energy $\Delta \varepsilon$ is added to a thin slab of atoms at $z=-L_{z} / 4$. Likewise, at each MD time step energy $\Delta \varepsilon$ is subtracted from a thin slab at $z$ $=+L_{z} / 4$. This results in two identical thermal currents, $J_{z}$, along the positive and negative $z$ axes, as labeled. The two crystallographically equivalent grain boundaries present in the cell at $z=-L_{z} / 2$ and $z=0$ are labeled as $\mathrm{GB}_{1}$ and $\mathrm{GB}_{2}$.

at $z=L_{z} / 4$, thereby conserving the total energy at all times. The resulting thermal currents $J_{z}$ and their directions are indicated in Fig. 1. The simulation cell also contains two GBs, located at $z=0$ and $z= \pm L_{z} / 2$. The latter GB is actually located along one face of the periodically repeated simulation cell; although this in no way affects the system's evolution, it means that the two regions close to the GB actually appear at opposite ends of the figures. Because we know the exact value of the thermal current flowing in the simulation cell, we only need to compute the temperature discontinuity at each GB and use Eq. (1) to determine $\sigma_{K}$. Throughout this article, we used the well-tested interatomic potential due to Stillinger and Weber ${ }^{17}(\mathrm{SW})$ with an MD time step of 0.55 fs.

The three-dimensionally periodic simulation cell used throughout, and sketched in Fig. 1, contains two identical grains that are misoriented with respect to each other by a twist rotation about the common GB-plane normal (defining the $z$ direction). Because of the periodic border condition in the $z$ direction, the simulation cell thus contains two crystallographically identical twist boundaries, characterized by the twist angles $\phi$ and $-\phi$, respectively. Because of the periodicity in the $x$ and $y$ directions, both boundaries are infinitely extended within the GB $(x-y)$ plane. As model systems we choose three rather different grain boundaries. The wellstudied (100) $\phi=43.60^{\circ}$ (so-called $\Sigma 29$ ) twist GB in silicon is generated by a twist rotation about the $\langle 100\rangle$ GB-plane normal by the twist angle $\phi=43.60^{\circ}$. Its relatively large, square planar unit cell contains $\Sigma=29$ atoms per (001) plane; its planar unit cell area is therefore 29 times larger than the primitive planar unit cell of a perfect-crystal (100) plane, i.e., prior to the $\langle 100\rangle$ twist rotation. This was chosen as a representative high-angle, high-energy GB with $E_{\mathrm{gb}}=1.32 \mathrm{~J} / \mathrm{m}^{2} .{ }^{18}$ For a low-angle, intermediate-energy GB, we chose the (001), $\phi=11.42^{\circ}(\Sigma 101)$ boundary, which has an energy of $E_{\mathrm{gb}}=0.91 \mathrm{~J} / \mathrm{m}^{2}{ }^{18}{ }^{18}$ Finally, for a high-angle, low-energy GB we selected the (111), $\phi=42.10^{\circ}(\Sigma 31)$ boundary, which has an energy of $E_{\mathrm{gb}}=0.64 \mathrm{~J} / \mathrm{m}^{2}{ }^{18}$ By studying three rather very different grain boundaries, we will be able elucidate the effect of structure and energetics on the Kapitza conductance and phonon scattering properties.
The structures of these grain boundaries differ from each other considerably. The (001) planes in Si are the third most widely spaced with a spacing of $0.25 a$. The $(001) \Sigma 29$, representative of a large number of high-energy GBs, consists of a region of approximately $2 \AA$ thickness that is highly disordered; indeed, by some measures it appears amorphous. ${ }^{18}$

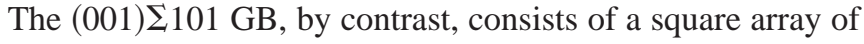
dislocations, separated by regions of perfect crystal. Analysis of their structure shows that these dislocation cores are also highly disordered. ${ }^{18}$ By contrast, the (111) planes are the most widely spaced. As a consequence, even high-angle GBs on this plane, such as the $\phi=42.10^{\circ} \Sigma 31$, have both relatively low energies and are characterized by a high degree of structural order.

To obtain system-size independent results, it is necessary that the simulation cell is quite long in the $z$ direction. Except for specific cases discussed below, the size in the $x-y$ plane was set to be the minimum consistent with the periodicity of the GB, i.e., 29 times, 31 times, and 101 times larger than that of the perfect crystal for the three GBs. The system

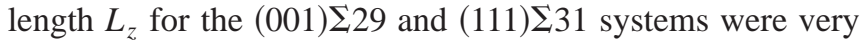
similar, being 223 and $224 \mathrm{~nm}$, respectively. Due to the larger dimension in the $x-y$ plane, the length $L_{z}$ of the (001) $\Sigma 101$ was set to be only $114 \mathrm{~nm}$. To generate the correct ground state structure of the grain boundaries, we used a high-temperature melting and annealing procedure used in a previous study of silicon GBs. ${ }^{18}$ The only difference from Ref. 18 was that, in this work, both GB regions were subject to the high-temperature melting and annealing procedure, and therefore were equivalent. After the high-temperature melting and annealing, the final ground state structure was obtained by relaxing the atoms to the $T=0 \mathrm{~K}$ ground state.

The resulting temperature profiles are shown in Fig. 2. For each case, the energy added and removed at each MD time step was chosen to result in a thermal-current density of $J=15.36 \mathrm{GW} / \mathrm{m}^{2}$. It is immediately apparent from Fig. 2 that the temperature discontinuity for the (001) $\Sigma 29$ boundary is

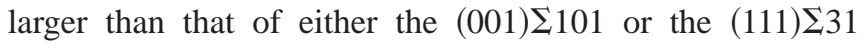
boundaries. From this we can conclude that the (001) $\Sigma 29$ boundary has the lowest Kapitza conductance. From the computed temperature discontinuity $\Delta T$ and the thermal current $J_{z}$, we use Eq. (1) to determine the value of $\sigma_{\mathrm{K}}$. The resulting values of the Kaptiza conductance are shown in Table I. Since the simulations of Maiti, Mahan, and Pantelides ${ }^{10}$ for two symmetric tilt GBs in silicon were determined using a very similar method and also for the SW potential, these results may be compared with ours. These are also included in Table I. It is remarkable that, in spite of the wide variety of structures considered, the values of $\sigma_{K}$ for the five different GBs are so very similar. While our results suggest that the amount of disorder in the grain boundary region can have a significant effect, it is apparent that $\sigma_{K}$ for the strongly disordered (001) $\Sigma 29$ boundary is actually very close to the results of Maiti and co-workers ${ }^{10}$ for the tilt GBs, which have a high degree of structural order. While this may suggest a difference between tilt and twist GBs, more systematic data are required before a definitive statement can be made. Finally, it is interesting to note that the values in Table I are rather close to the experimental 

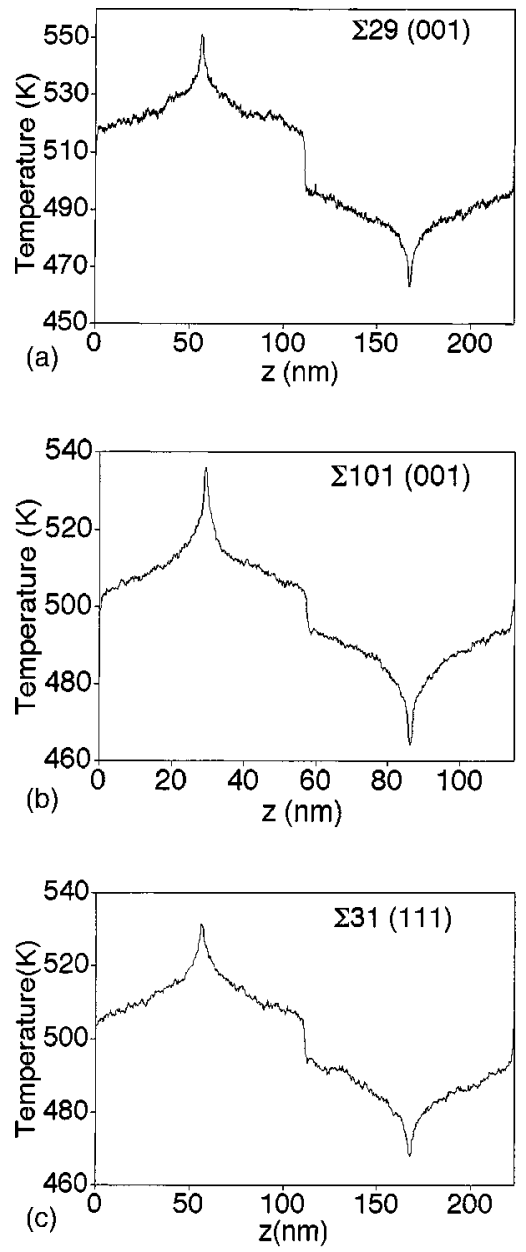

FIG. 2. Temperature profiles in the presence of an applied thermal current for the (a) (001) $\phi=43.60^{\circ} \Sigma 29$, (b) (001) $\phi=11.42^{\circ} \Sigma 101$, and (c) (111) $\phi=42.10^{\circ} \Sigma 31$ grain-boundary systems. In each case the location of the grain boundary coincides with the discontinuous jump in the temperature profile. The applied thermal current is the same in each case.

results of Yang et al..$^{9}$ for yttria-stabilized zirconia, and therefore quite different in magnitude from the values reported by Nan and Birringer. ${ }^{8}$

Grain boundaries have two effects on thermal transport. First, as discussed above they introduce structural disorder that leads to phonon scattering even when amplitude of the atomic displacements is small enough that the system remains harmonic. Second, the structural disorder itself changes the dynamical behavior of the atoms at the interface.

TABLE I. Values of the Kapitza conductance for the three twist grain boundaries studied in this work at $T=500 \mathrm{~K}$. For comparison, we also include in this table values of $\sigma_{K}$ at $575 \mathrm{~K}$ taken from Maiti and co-workers (see Ref. 10) for two symmetric tilt grain boundaries (STGBs).

\begin{tabular}{|c|c|c|c|}
\hline Grain boundary & $E_{\mathrm{gb}}\left(\mathrm{J} / \mathrm{m}^{2}\right)$ & $\begin{array}{c}\sigma_{K}\left(\mathrm{GW} / \mathrm{m}^{2} \mathrm{~K}\right) @ \\
500 \mathrm{~K}\left(^{*}\right) \\
\text { and } 575 \mathrm{~K}\left(^{\dagger}\right)\end{array}$ & $\begin{array}{c}\sigma_{K}\left(\mathrm{GW} / \mathrm{m}^{2} \mathrm{~K}\right) @ \\
1000 \mathrm{~K}\end{array}$ \\
\hline (001) $\theta=43.60^{\circ} \Sigma 29$ & 1.32 & $0.80 \pm 0.10^{*}$ & $0.99 \pm 0.10$ \\
\hline (001) $\theta=11.42^{\circ} \Sigma 101$ & 0.91 & $1.63 \pm 0.20 *$ & $1.95 \pm 0.20$ \\
\hline (111) $\theta=42.10^{\circ} \Sigma 31$ & 0.64 & $1.42 \pm 0.20^{*}$ & $1.46 \pm 0.20$ \\
\hline$(310) \sum 5$ STGB & & $0.9^{\dagger}$ & $\cdots$ \\
\hline (510) $\Sigma 13$ STGB & & $0.8^{\dagger}$ & $\cdots$ \\
\hline
\end{tabular}

As a result, anharmonic scattering will be more important even for relatively small atomic displacements. In order to elucidate the effects of anharmonicity, we studied the effect of temperature on the thermal conductivity. We first recall that our previous simulations of bulk Si showed strong temperature dependence, with the thermal conductivity, $\kappa$, decreasing from $119 \mathrm{~W} / \mathrm{mK}$ at $500 \mathrm{~K}$ to $65 \mathrm{~W} / \mathrm{mK}$ at $1000 \mathrm{~K}$. By contrast, we see in Table I that the interfacial conductance, although rather insensitive to temperature, does increase slightly with increasing temperature, i.e., the opposite of the trend in the bulk thermal conductivity. However, the differences are fairly small and still within the error inherent in the calculation of $\sigma_{K}$. This suggests that, while anharmonic effects may play some role, they are not particularly important at least within this temperature range. As a result, it is likely that the computed values of $\sigma_{K}$ in this temperature range are due mostly to harmonic scattering at the interface. On the other hand, it is possible that the amount of anharmonic scattering at the boundary occurring at $T=500 \mathrm{~K}$ is already rather strong, and increasing the temperature to $T$ $=1000 \mathrm{~K}$ does not substantially increase the amount of anharmonic scattering. In order to systematically address the issue of the importance of anharmonic scattering, it will be necessary to simulate the Kapitza conductance over a wider range of temperatures than we report here.

Finally, we have also elucidated the finite-size effects. As we previously mentioned, the simulation cell for (001) 1101 GB was chosen to be shorter than the other systems due to its larger dimension in the $x-y$ plane. It is therefore important to determine if the results shown in Table I depend systematically on $L_{z}$. To answer this question, we have simulated the (001) $\Sigma 29$ boundary system with a length $L_{z}$ of $114 \mathrm{~nm}$, the same as that used for the $\Sigma 101 \mathrm{~GB}$ and about one half the length of the system used to generate the data in Table I. We find values for $\sigma_{K}$ of $0.81 \pm 0.10$ $\mathrm{GW} / \mathrm{m}^{2} \mathrm{~K}$ at $T=500 \mathrm{~K}$ and $0.87 \pm 0.10 \mathrm{GW} / \mathrm{m}^{2} \mathrm{~K}$ at $T$

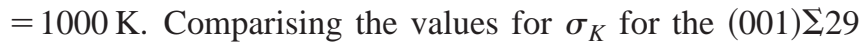
boundary computed using the larger system shown in Table I, we see that changing $L_{z}$ appears to have very little effect. This indicates that our results for the (001) $\sum 101$ system can be directly compared to the results for the other GBs in spite of the differences in $L_{z}$.

In this section we have presented the results of MD simulations used to determine the values of $\sigma_{K}$ for different twist grain boundaries in silicon. We observed that the highenergy, disordered (001)乏29 grain boundary has a smaller value of $\sigma_{K}$ when compared to the lower-energy, more ordered grain-boundary systems. This suggests that disorder at the grain boundary plays an important role in the scattering of lattice waves. However, we do not yet have any understanding of the microscopic mechanisms involved. In the next section, we will address this issue by scattering wave packets of lattice vibrations of well-defined frequency and polarization from grain boundaries.

\section{WAVE-PACKET SCATTERING AT GRAIN-BOUNDARY INTERFACES}

The simulations presented in the last section allowed us to make a direct comparison of $\sigma_{K}$ for different grain bound- 
aries. However, this did not result in a direct understanding of the underlying scattering mechanisms. Furthermore, because the simulations were classical, all modes were equally excited, and thus these simulations cannot be used to investigate any possible dependence of the interfacial scattering on phonon frequency. To address the problem of interfacial phonon scattering, we describe in this section the results of simulations of the scattering of phonon wave packets from

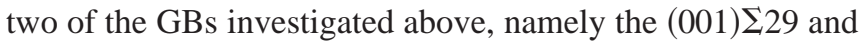
the (001) $\Sigma 101$. The basic idea, as described in detail below, is to generate well-defined wave packets of lattice vibrations, using the normal modes of the bulk perfect crystal, and to allow them to interact and scatter from an interface. We have previously developed this method and demonstrated its usefulness for studying the frequency-dependent scattering from simple coherent interfaces. ${ }^{19}$ In this section, we show how this approach is applied to the study of grain-boundary phonon scattering.

We form the phonon wave packets from a linear combination of vibrational eigenstates using the procedure described previously. ${ }^{19}$ In brief, the initial atomic displacements are described in terms of a linear combination of eigenstates

$$
u_{i l \mu}(0)=\sum_{\lambda \mathbf{k}} a_{\lambda \mathbf{k}} \varepsilon_{i \mu \lambda k} \exp \left(i \mathbf{k} \cdot \mathbf{R}_{l}\right) .
$$

Here, $u_{i l \mu}(0)$ represents the $\mu$ th Cartesian component of the displacement for atom $i$ in the unit cell labeled by $l$. The polarization vector, $\varepsilon_{i \mu \lambda \mathbf{k}}$, of the normal mode with wave vector $\mathbf{k}$ in band $\lambda$, is determined by diagonalizing the dynamical matrix of the bulk perfect crystal. The $a_{\lambda \mathbf{k}}$, which determine the amplitude of the normal modes, are chosen to result in vibrational wave packets that are localized in both real space and wave vector space. ${ }^{19}$ This is done by localizing the chosen vibrational mode using a Gaussian envelope. Given these resulting displacements, the coefficients $a_{\lambda \mathbf{k}}$ are determined by transforming into the space defined by the normal modes. In the absence of any scattering, the time dependence of the atomic displacements is exactly given by

$$
u_{i l \mu}(t)=\sum_{\lambda \mathbf{k}} a_{\lambda \mathbf{k}} \varepsilon_{i \mu \lambda \mathbf{k}} \exp \left(i \mathbf{k} \cdot \mathbf{R}_{l}-i \omega_{\lambda \mathbf{k}} t\right),
$$

where $\omega_{\lambda \mathbf{k}}$ is the angular frequency of the normal mode with wave vector $\mathbf{k}$ in band $\lambda$. In other words, in the absence of any scattering, the $a_{\lambda \mathbf{k}}$ are time-independent constants, and the shape of the initial wave packet evolves in real space only very slowly due to phonon dispersion.

The time dependence of Eq. (3) assumes a periodic perfect crystal lattice of infinite extent. With the introduction of grain boundaries, the periodicity of the crystal lattice is destroyed, and as a result the time dependence of the atomic displacements given by Eq. (3) will not apply. This means that the $a_{\lambda \mathbf{k}}$ are no longer time independent but will change as a result of scattering at the grain-boundary interface. As was previously discussed, this scattering results in a finite value of the Kapitza conductance. In terms of the normal modes of the bulk lattice, the Kapitza conductance can be written as

$$
\sigma_{K}(T)=\frac{1}{\Omega} \sum_{\lambda \mathbf{k}} \hbar \omega_{\lambda \mathbf{k}} \frac{\partial n\left(\omega_{\lambda \mathbf{k}}, T\right)}{\partial T} \frac{\partial \omega_{\lambda \mathbf{k}}}{\partial k_{z}} \alpha_{\lambda \mathbf{k}},
$$

where $\Omega$ is the system volume, $n\left(\omega_{\lambda \mathbf{k}}, T\right)$ is the Bose occupation factor at temperature $T$, and $\alpha_{\lambda \mathbf{k}}$ is the energy transmission coefficient for the interface. The $\alpha_{\lambda \mathbf{k}}$ is defined as the fraction of incident energy in a mode defined by phonon branch $\lambda$ and wave vector $\mathbf{k}$ is transmitted across the interface. Equation (3) suggests that, given an understanding of the phonon dispersions of the bulk perfect crystal, the value of $\sigma_{K}$ can be determined if the $\alpha_{\lambda \mathbf{k}}$ can be computed for each mode.

In passing, we note that Eq. (4) results in a finite conductance even in the case where there is no boundary and $\alpha_{\lambda \mathbf{k}}=1$ for all $\lambda$ and $\mathbf{k}$. However, this apparent paradox has been resolved by Pettersson and Mahan. ${ }^{5}$ They showed that in the case of an imagined interface with $\alpha=1$, the theory reduces to the standard theory of thermal conduction when there is no boundary present.

To gain insight into the scattering process, the atomic positions after the scattering event can be analyzed in terms of the normal modes of the bulk perfect crystal by Fourier transforming the atomic positions using the eigenvectors of the bulk perfect crystal. In other words, given a set of atomic displacements $u_{i l \mu}\left(t=t_{f}\right)$ at final time $t_{f}$, we can determine the amplitude $a_{\lambda \mathbf{k}}$ of a given mode from

$$
a_{\lambda \mathbf{k}}=\sum_{i l \mu} \varepsilon_{\lambda \mathbf{k} i \mu}^{*} u_{i l \mu}\left(t=t_{f}\right) \exp \left(-i \mathbf{k} \cdot \mathbf{R}_{l}\right) .
$$

Given the amplitude of each normal mode, it is possible to directly compute the amount of energy contained in a given mode. By including only atoms left or right of the grain boundary, we can study the reflected and transmitted waves separately.

For the current problem, we have chosen to study the scattering of incident LA wave packets from the (001) $\Sigma 29$ and (001) ¿101 grain boundaries. We chose LA modes because they are the most important branch for thermal transport. In particular, we consider the case where the incident wave packet has a wave vector perpendicular to the interface. In other words, the wave vector of the incident phonon is parallel to the $z$ axis: $k_{z} \neq 0$, whereas $k_{x}=0$ and $k_{y}=0$. For our phonon scattering simulations, we required simulation cells considerably longer than those needed for the thermalconductivity calculations. In both cases, the simulation cell was at least 1000 unit cells in length, corresponding to about $0.55 \mu \mathrm{m}$.

To more fully understand the scattering process it is useful to analyze the dynamics at the microscopic level. In Figs. 3(a) and 3(b) we show snapshots at different times of the $z$ displacement of the atoms as a function of position along the $z$ direction in the simulation cell for two different incident wave vectors. Figure 3(a) shows the scattering from the (001) 229 GB of a phonon wave packet with a very long average wavelength, $40 a_{0}$, corresponding to an average frequency of $0.37 \mathrm{THz}$ and a wave vector of $k_{z}$ $=0.025\left(2 \pi / a_{0}\right)$. It is apparent that this wave packet is almost unaffected by the presence of the grain boundary, and nearly all the incident energy is transmitted to the region $z$ 

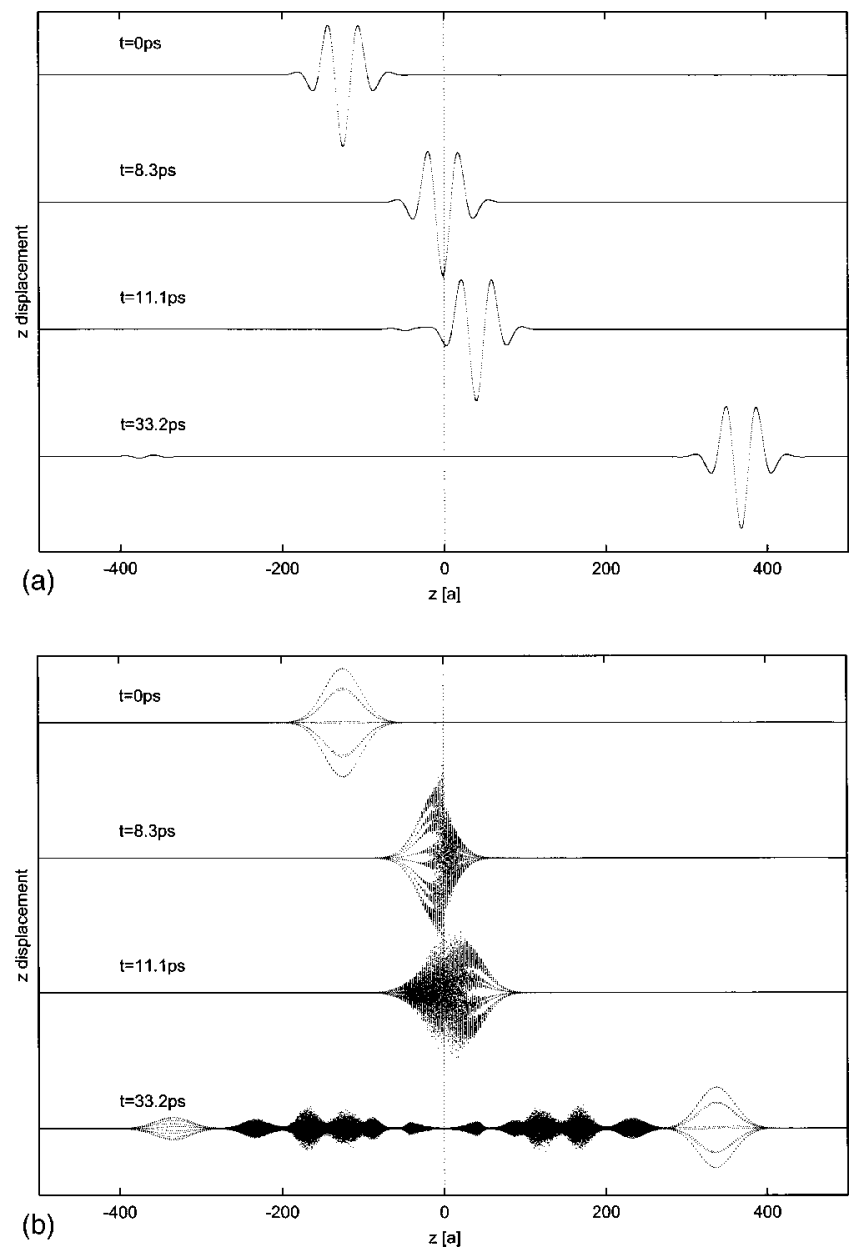

FIG. 3. Snapshots of the $z$ displacement of atoms for the case of an LA wave-packet scattering from the (001) $\Sigma 29$ grain boundary. In (a) we show the scattering of an LA wave packet with $k_{z}=0.025\left(2 \pi / a_{0}\right)$, and in (b) for a wave packet with $k_{z}=0.400\left(2 \pi / a_{0}\right)$. The vertical dotted line at $z=0$ indicates the location of the grain boundary.

$>0$. By contrast, the wave packet shown in Fig. 3(b) with an average wavelength of $2.5 a_{0}$ and frequency and wave vector of $5.81 \mathrm{THz}$ and $k_{z}=0.400\left(2 \pi / a_{0}\right)$ is strongly scattered by the interface, with a significant amount of the total energy being reflected back to the region $z<0$. The spread in real space of the atomic displacements seen in Fig. 3(b) at $33.2 \mathrm{ps}$ suggests that a significant amount of the incident energy has been scattered into modes different from the incident wave packet.

In Fig. 4 we show the frequency dependence of the transmission coefficient $\alpha$ for wave packets comprised of LA modes scattering from the (001) $\Sigma 29$ grain boundary. These data are compiled from an analysis of snapshots such as those at the latest time in Figs. 3(a) and 3(b). Also shown in Fig. 4 is the reflection coefficient $\beta$, which is defined to be the fraction of the incident energy that is reflected from the grain boundary to the region $z<0$. Since all of the incident energy is either transmitted to the region $z>0$ or reflected to the region $z<0, \alpha+\beta=1$ for each incident frequency.

As we have already seen, along with reflection of the incident wave, there is significant mode conversion for both the transmitted and reflected waves. Because the simulation

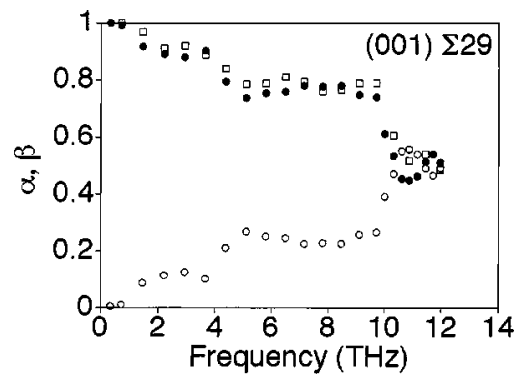

FIG. 4. Frequency dependence of the energy transmission coefficient, $\alpha$ (filled circles), and reflection coefficient, $\beta$ (open circles), for incident LA wave packets scattered from the (001) $\Sigma 29 \mathrm{~GB}$ in Si. Since all energy is either reflected from or transmitted through the boundary, $\alpha+\beta=1$. The squares denote $\alpha$ for a simulation cell containing $2 \times 2$ cells of the (001) $\Sigma 29$ grain boundary system.

cell is finite, the possible final states depend on the size of the simulation cell. This suggests that the results of Fig. 4 may depend on the size of the simulation cell. To determine the dependence of our results on system size, we also show results for the energy transmission coefficient of a simulation cell which is twice as large in the $x-y$ plane. It thus contains four CSL planar unit cells of the (001) 29 grain-boundary system. By doubling the cell in the $x$ and $y$ directions, we are increasing the number of possible vibrational states that can be excited by a factor of 4 . A comparison of these two systems in Fig. 4 indicates the transmission coefficient is almost identical for the larger unit cell. Together with our previous demonstration that the transmission coefficient does not depend on $L_{z}$, these data establish the system-size independence of our results.

It is apparent from Fig. 4 that the scattering depends strongly, but not smoothly, on the frequency of the incident wave packet. For very low frequencies, i.e., long wavelengths, the transmission coefficient is very close to unity, in agreement with the AMM. However, the fraction of the energy transmitted decreases as the frequency of the incident wave packet is increased; indeed for very high frequencies the transmission coefficient is close to 0.5 , i.e., it is close to the value expected from the DMM.

To analyze this transition from AMM behavior to DMM behavior, we have analyzed the reflected and transmitted waves in more detail. To describe the component of the wave vector parallel to the interface, we first define a quantity $k_{x y}=\sqrt{k_{x}^{2}+k_{y}^{2}}$. For the incident wave packet, only modes with $k_{x y}=0$ are used. In Fig. 5, we break up the transmitted wave from Fig. 3(b) into three components: an unscattered LA mode, i.e., a mode with the same $\mathrm{k}$ vector as the incident wave packet, a scattered LA mode which has $k_{x y}>0$, and TA modes; none of the incident LA modes has sufficient energy to create optical modes, so they need not be considered here. From Fig. 5 we see that much of the incident wave packet is not scattered and remains in LA modes with $k_{x y}=0$. This unscattered wave packet is closely followed by wave packets comprised of LA modes with nonzero values of $k_{x y}$. Finally, a significant amount of energy is found in TA modes. The positions of the wave packets can be understood in terms of the $z$ component of the group velocities of the different modes. Although there is a significant amount of mode con- 


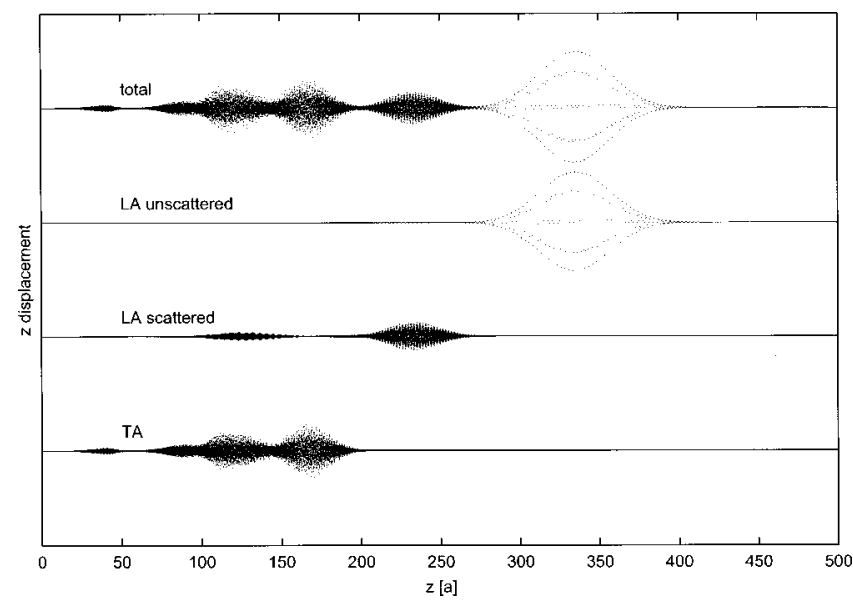

FIG. 5. Analysis of the transmitted wave packets for a wave packet with $k_{z}=0.400\left(2 \pi / a_{0}\right)$ incident on the $(001) \Sigma 29 \mathrm{~GB}$. The displacements in this picture correspond to those in Fig. 3(b) at $t=33.2$ ps. At the top, we show the total atomic displacements. In the next panel below, we show only the components of the wave that correspond to LA modes with $k_{x y}=0$. Because these modes are the same as those that comprised the incident wave packet, this part of the transmitted wave is unscattered. We next show the LA modes that are scattered, i.e., those modes that have $k_{x y}>0$. Finally, in the bottom panel we show the displacements that are just due to TA modes.

version, we find that only modes with vibrational frequencies equal to the frequency of the incident wave packet are excited, corresponding to perfectly elastic scattering.

The scattering event can be further analyzed by studying the fraction of energy scattered into various modes using Eq. (5). We first show in Fig. 6 the fraction of energy transmitted into LA modes with $k_{x y}=0$. For the waves transmitted to the region $z>0$, this represents the portion of the incident energy that is not scattered by the grain boundary. We see that a significant fraction of the energy is in fact not scattered by the grain boundary below a frequency of about $9 \mathrm{THz}$. However, for frequencies above $9 \mathrm{THz}$, most of the incident energy is scattered.

For the case of scattering that involves normal modes different from the incident LA mode, it is important to consider the density of states (DOS) for the perfect crystal grain. For the (001) $\Sigma 29$ system, the total DOS is shown in Fig. 7. By comparing to the DOS for an infinite system shown in the inset of Fig. 7, it is clear that much of the structure in Fig. 7 is due to the finite size of the simulation cell. We analyze the

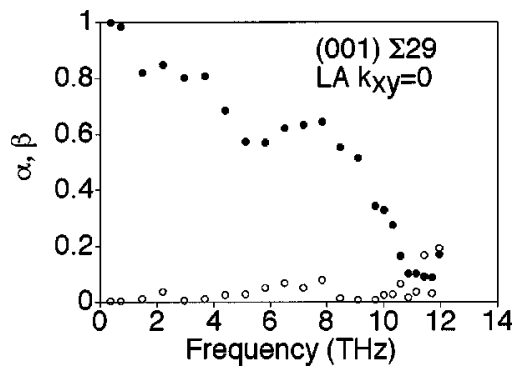

FIG. 6. The transmission coefficient $\alpha$ and the reflection coefficient can be understood in terms of the contributions of the various final states. Shown for incident LA wave packets scattered from a $(001) \Sigma 29$ grain boundary are the fraction of the incident energy that is transmitted (filled circles) and reflected (open circles) into LA modes with $k_{x y}=0$.

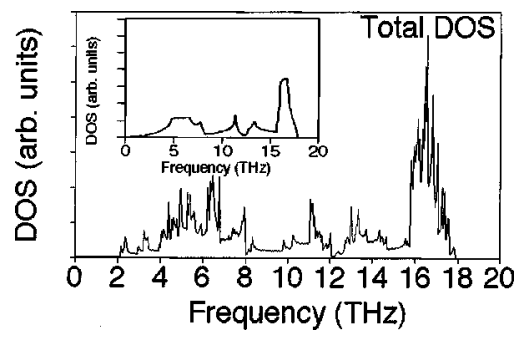

FIG. 7. Total density of states (DOS) for a bulk perfect crystal with the size and orientation of one of the two grains in the simulation cell used to study the (001) $\Sigma 29$ grain boundary. The inset shows the total DOS for an infinite bulk perfect crystal.

scattering into TA modes and LA modes with $k_{x y}>0$ in Fig. 8. In Fig. 8(a), we see that significant amounts of the incident wave packet can be transmitted and reflected into TA modes. In Fig. 8(b) we show the contribution to the total DOS (see Fig. 7) that is due to the TA modes. It is clear that the scattering into TA modes can happen only for incident frequencies where there is a finite DOS of TA modes. Figure 8(c) shows that the energy transmitted and reflected into LA modes with $k_{x y}>0$ can be significant, especially for frequencies above $8 \mathrm{THz}$. As with the TA modes, it can be seen from Fig. 8(d) that the amount of energy scattered into these modes depends strongly on the DOS due to these modes.

For the scattering of LA wave packets from the (001) $\Sigma 101$ boundary, the picture is rather different. In Fig. 9 we show the transmission and reflection coefficients for this boundary. Comparing Fig. 9 to Fig. 4, it is apparent that a larger fraction of the incident energy is transmitted to the region $z>0$. Furthermore, Fig. 10 shows that much of the transmitted energy remains in LA modes with $k_{x y}=0$, and hence is not scattered by the grain boundary. For all of the
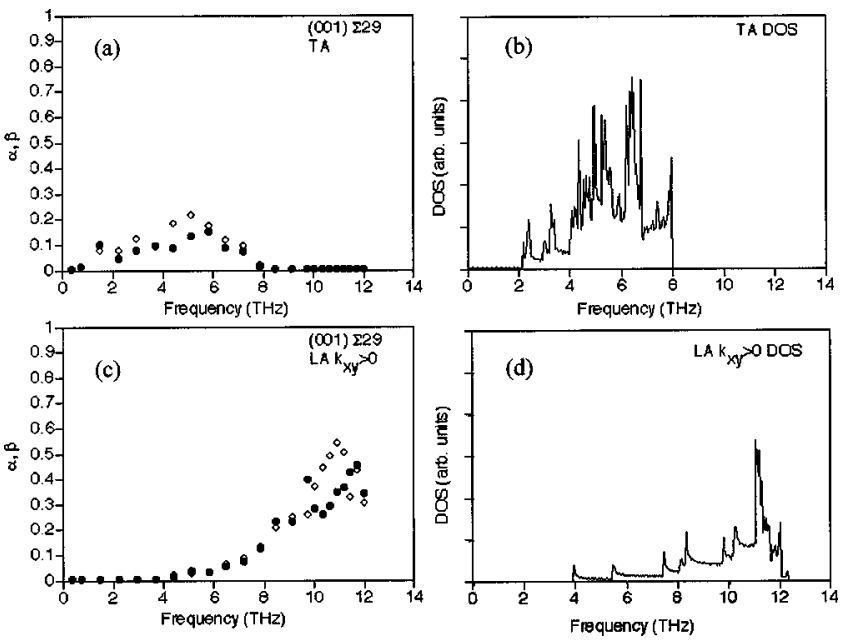

FIG. 8. The final states excited after the scattering event depend strongly on the DOS at the frequency of the incident wave packet. For scattering from the (001) $\Sigma 29$ grain boundary, the incident LA wave packet can excite TA modes and also LA modes with $k_{x y}>0$. We show in (a) the fraction of incident energy transmitted (filled circles) and reflected (open circles) into TA modes. In (b) we show the contribution to the total DOS (see Fig. 7) due to TA modes. The fraction of the incident energy transmitted (filled circles) and reflected (open circles) into LA modes with $k_{x y}>0$ is shown in (c), with the contribution to the DOS of these modes shown in (d). 


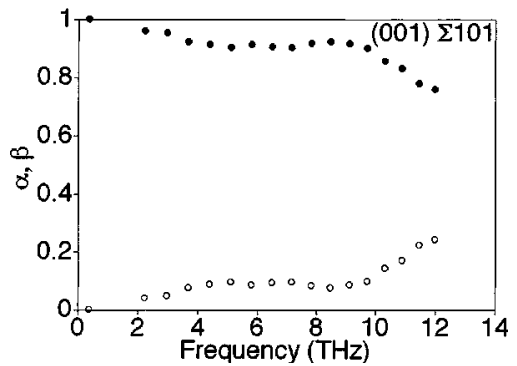

FIG. 9. Frequency dependence of $\alpha$ (filled circles) and $\beta$ (open circles) for incident LA wave packets scattered from a (001) $\Sigma 101$ grain boundary.

frequencies shown in Fig. 10, at least $60 \%$ of the incident energy is transmitted without scattering.

Consistent with this, in Fig. 11 we see that only rather small amounts of energy are transmitted and reflected into TA modes and LA modes with $k_{x y}>0$. As with the (001) $\Sigma 29$ boundary, the scattering into these modes depends on the presence of a finite DOS at the frequency of the incident wave packet. Comparing Fig. 11 with the results for the (001) 229 boundary system shown in Fig. 8, we can immediately see that the amount of mode conversion is significantly less for the (001) $\Sigma 101$ boundary. We believe this is due to the more ordered atomic-scale structure present at the (001)乏101 boundary.

The results presented here for the scattering of boundaries show in detail the mechanism of phonon scattering from grain boundaries. Consistent with our expectations from the MD simulations of the Kaptiza conductance presented in Sec. II, we found here that the (001) $\Sigma 29$ boundary scattered phonons more strongly than the (001) $\sum 101$ boundary. We believe that this difference arises from the more disordered structure present in the (001) 29 boundary. In both cases, in the limit of very low frequency, the wave packet passed through the grain boundary with essentially no scattering. By contrast, as the frequency of the incident wave packet was increased, significant mode conversion was found. Taken together, these results suggest that the scattering becomes more diffuse as the frequency of the incident wave is increased. We will address this point in the final section, where our results will be discussed in the context of the AMM and DMM.

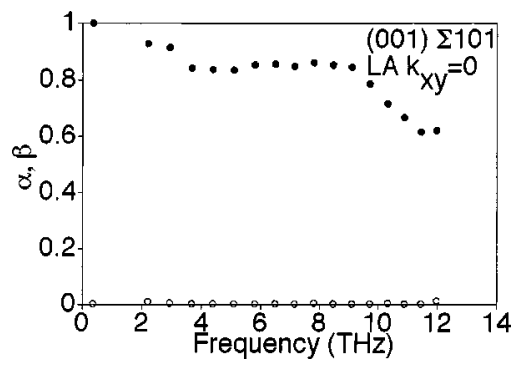

FIG. 10. For incident LA wave packets scattering from the (001) $\Sigma 101$ grain boundary, the fraction of the incident energy that is transmitted (filled circles) and reflected (open circles) into LA modes with $k_{x y}=0$.
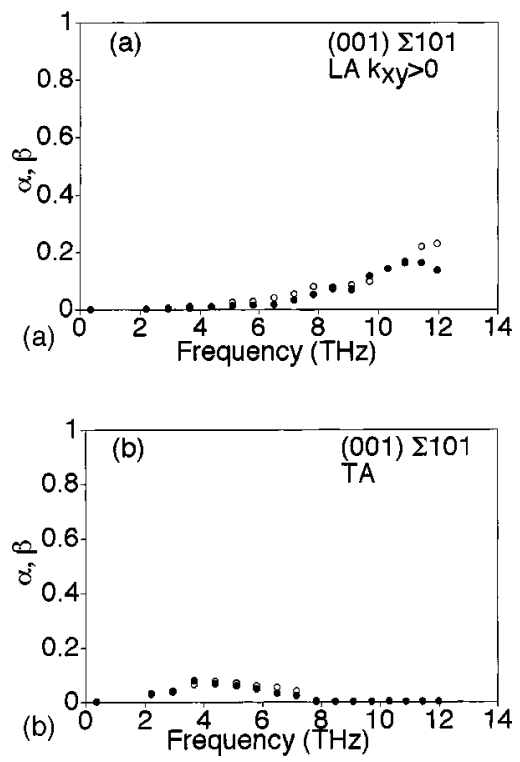

FIG. 11. LA modes scattered from the (001) $\Sigma 101$ grain boundary: (a) the fraction of the energy transmitted (filled circles) and reflected (open circles) into LA modes with $k_{x y}>0$; (b) the fraction of the incident energy that is transmitted (filled circles) and reflected (open circles) into TA modes.

\section{DISCUSSION AND CONCLUSIONS}

Using MD simulation, we have directly determined the Kapitza conductance for three different twist grain boundaries in silicon. Our results indicate that the structure and energy of the grain boundary is important in determining the Kapitza conductance. In particular, we found that the highly disordered (001) 29 grain boundary has a significantly lower Kapitza conductance than the more ordered, lowerenergy $(001) \Sigma 101$ and (111) $\Sigma 31$ grain boundaries.

It is interesting to compare the values obtained here for the Kapitza conductance of twist grain boundaries in silicon to other results found in the literature. We have already shown in Table I that our results are very similar to MD simulation results for symmetrical tilt grain boundaries in $\mathrm{Si}^{10}{ }^{10}$ Furthermore, the magnitude of the Kapitza conductance calculated here appears to be similar to recent experimental results for grain boundary Kapitza conductance in yttriastabilized zirconia. ${ }^{9}$ By contrast, very low values for the Kapitza conductance of grain boundaries have been seen in a few thermoelectric alloys. ${ }^{8}$ For example, the Kapitza conductance of grain boundaries in the $p$-type alloy $\mathrm{Bi}_{2} \mathrm{Te}_{3} / \mathrm{Sb}_{2} \mathrm{Te}_{3}$ was found to be $0.7 \times 10^{4} \mathrm{GW} / \mathrm{m}^{2} \mathrm{~K},{ }^{8}$ or four orders of magnitude smaller than the Kapitza conductances found here. It is also interesting to compare our results to experimental measurements of the Kapitza conductance of interfaces between different materials. For example, the roomtemperature Kapitza conductance of a diamond/ $\mathrm{Pb}$ interface was found by Stoner and Maris ${ }^{7}$ to be about $0.03 \mathrm{GW} / \mathrm{m}^{2} \mathrm{~K}$, significantly lower than the results found here for $\mathrm{Si}$ grain boundaries. That diamond/ $\mathrm{Pb}$ interfaces have a relatively small Kapitza conductance is not surprising since diamond and $\mathrm{Pb}$ have very different acoustic properties. However, there are several examples of interfaces between different materials that have a Kapitza conductance very similar in magnitude to the results found in this article for $\mathrm{Si}$ grain 
boundaries: for example, the room-temperature Kapitza conductance of epitaxial TiN/oxide interfaces was found to be about $0.7 \mathrm{GW} / \mathrm{m}^{2} \mathrm{~K}{ }^{6}$

By using wave-packet dynamics, the underlying scattering mechanisms responsible for the Kapitza conductance can be understood in detail. In Sec. III, we presented a detailed analysis of the scattering in terms of the normal modes of the bulk perfect crystal. This analysis showed that the scattering depends strongly on the frequency of the incident wave and also the structure and energy of the grain boundary. Consistent with the MD results for the Kapitza conductance, we

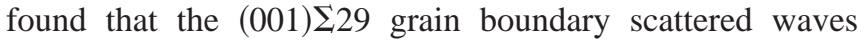
more strongly than the (001) $\sum 101$ grain boundary.

The strong frequency dependence of the energy transmission coefficient seen in Figs. 4 and 9 was not present in early analytical work ${ }^{11}$ because the analytical derivation assumes that phonon scattering is due only to the strain field of the grain boundary, and not to the atomic-scale structure of the interface itself. Because analytical approaches do not treat the details of the atomic structure of the boundary, they cannot be expected to apply to the limit of small wavelength and high frequency. The results of Figs. 4 and 9 suggest interesting consequences for the temperature dependence of the Kapitza conductance below the Debye temperature of a material. The frequency-independent energy transmission coefficient suggested by analytical methods results in a Kapitza conductance that increases with increasing temperature as $T^{3}$ for temperatures well below the Debye temperature. However, if in fact the energy transmission coefficient is a decreasing function of frequency as our results suggest, it is likely that the Kapitza conductance will increase more slowly than $T^{3}$.

It is useful to compare our results to the predictions of the AMM and DMM. Since the AMM deals only with the mass density and elastic constants, it predicts that the grain boundaries studied here should completely transmit all the incident energy, i.e., $\alpha=1$. We see from Figs. 4 and 9 that this indeed is the case in the limit of $\omega \rightarrow 0$. However, as the frequency of the incident wave packet increases, the energytransmission coefficient becomes significantly less than unity. For a very disordered interface, such as the (001) $\Sigma 29$ grain boundary, it is conceivable that the DMM may be a good model for the scattering. However, our results suggest that this is probably not the case for much of the phonon spectrum. First of all, when applied to GB scattering the DMM results in a value for the energy transmission coefficient $\alpha$ of 0.5. As shown in Fig. 4, the energy transmission coefficient $\alpha$ is more than 0.5 for all but the highest frequencies. The results for the (001) $\sum 101$ grain boundary shown in Fig. 9 are significantly higher than 0.5 for the entire frequency range. Second, the amount of energy transmitted through the grain boundary without scattering is usually quite significant (see Figs. 6 and 10). By contrast, the DMM is predicated on the assumption that essentially all of the incident energy is scattered from the interface. This limit appears to be realized only for the very high frequency phonons $(>10 \mathrm{THz})$ incident on the (001) 229 grain boundary (see Fig. 6).
These considerations suggest that the AMM and DMM represent extreme limits of phonon scattering, and that in reality, for most of the phonon spectrum, the scattering lies somewhere in between these limits. For very long wavelength modes, the AMM appears to describe our results, even in the case of the disordered (001) $\Sigma 29$ grain boundary. As the frequency of the incident mode is increased, the scattering becomes progressively more diffuse, with the amount of diffuse scattering depending strongly on the amount of disorder at the grain boundary interface. In fact, our results also suggest that the diffuse limit is only realized for scattering from a very disordered interface.

One important question not fully addressed in this article is the importance of anharmonic effects on interfacial scattering. As noted earlier in Sec. III, the amplitude of the incident wave packet was always chosen to be small enough so that there were essentially no anharmonic effects. However, for a system at finite temperature, scattering due to anharmonicity is likely to be important. We would expect that for a $\mathrm{GB}$, anharmonicity would result in more scattering at the interface. This increased scattering would tend to cause the Kaptiza conductance to decrease with increasing temperature. If anharmonic effects are significant, this may result in more diffuse scattering than we found in Sec. III, and hence better agreement with the predictions of the DMM.

Another well-known effect of increasing the temperature is to increase the thermal disorder in the GB region; ${ }^{18}$ such disorder should result in more scattering and hence a lower value of the Kapitza conductance. This suggests that the predictions of the DMM may become more valid at higher temperatures.

Finally, we mention that the results of this article can be used to develop more realistic models of interfacial scattering for use in mesoscale simulation approaches. In a mesoscale approach, the energy is transported by particles localized in real space meant to represent localized phonon wave packets. ${ }^{20}$ To describe transport in a system with interfaces, rules are required to describe the scattering at interfaces. From the results presented in this article, one could develop a simple yet general model to describe grain-boundary scattering. Specifically, our results indicate that any model should have a transmission coefficient essentially equal to unity for long-wavelength phonons. The scattering in such a model should increase with increasing frequency, with the degree of scattering gradually approaching the DMM limit at least in the case of disordered grain boundaries. Such a model would represent an improvement over current approaches that are limited either to the extremes of the AMM or the DMM.

\section{ACKNOWLEDGMENT}

This work was supported by the U.S. Department of Energy, Office of Science, under Contract No. W-31-109-Eng38. P.K. was supported by NSF Grant No. DMR 134725.

\footnotetext{
${ }^{1}$ P. L. Kapitza, J. Phys. (Moscow) 4, 181 (1941).

${ }^{2}$ E. T. Swartz and R. O. Pohl, Rev. Mod. Phys. 61, 605 (1989).

${ }^{3}$ I. M. Khalatnikov, Zh. Eksp. Teor. Fiz. 22, 687 (1952).

${ }^{4}$ D. A. Young and H. J. Maris, Phys. Rev. B 40, 3685 (1989).
} 
${ }^{5}$ S. Pettersson and G. D. Mahan, Phys. Rev. B 42, 7386 (1990).

${ }^{6}$ R. M. Costescu, M. A. Wall, and D. G. Cahill, Phys. Rev. B 67, 054302 (2003).

${ }^{7}$ R. J. Stoner and H. J. Maris, Phys. Rev. B 48, 16373 (1993).

${ }^{8}$ C.-W. Nan and R. Birringer, Phys. Rev. B 57, 8264 (1998).

${ }^{9}$ H. S. Yang, G. R. Bai, L. J. Thompson, and J. A. Eastman, Acta Mater. 50, 2309 (2002).

${ }^{10}$ A. Maiti, G. D. Mahan, and S. T. Pantelides, Solid State Commun. 102, 517 (1997).

${ }^{11}$ P. G. Klemens, Proc. Phys. Soc., London, Sect. A 68, 1113 (1955).

${ }^{12}$ R. Kubo, Rep. Prog. Phys. 29, 255 (1966).
${ }^{13}$ P. K. Schelling, S. R. Phillpot, and P. Keblinski, Phys. Rev. B 65, 144306 (2002).

${ }^{14}$ J. Li, L. Porter, and S. Yip, J. Nucl. Mater. 255, 913 (1998).

${ }^{15}$ S. G. Volz and G. Chen, Phys. Rev. B 61, 2651 (2000).

${ }^{16}$ P. Jund and R. Jullien, Phys. Rev. B 59, 13707 (1999).

${ }^{17}$ F. H. Stillinger and T. A. Weber, Phys. Rev. B 31, 5262 (1985).

${ }^{18}$ P. Keblinski, S. R. Phillpot, and D. Wolf, J. Am. Ceram. Soc. 80, 717 (1997).

${ }^{19}$ P. K. Schelling, S. R. Phillpot, and P. Keblinski, Appl. Phys. Lett. 80, 2484 (2002).

${ }^{20}$ S. Mazumder and A. Majumdar, J. Heat Transfer 123, 749 (2001). 